\title{
Viral Genome Conformations and Contacts across Different Lifecycle Stages ${ }^{\dagger}$
}

\author{
Peter G. Stockley ${ }^{1, *}$, Nikesh Patel ${ }^{1}$, Emma L. Wroblewski ${ }^{1}$, Andrew J. P. Scott ${ }^{1}$, Carlos P. Mata ${ }^{1}$, \\ Rebecca Chandler-Bostock ${ }^{1}$, Amy M. Barker ${ }^{1}$, Reidun Twarock ${ }^{2}$, Sam Clark ${ }^{2}$ \\ and Richard J. Bingham ${ }^{2}$ \\ 1 Astbury Centre for Structural Molecular Biology, Faculty of Biological Sciences, University of Leeds, \\ Leeds LS2 9JT, UK; n.patel@leeds.ac.uk (N.P.); fbselw@leeds.ac.uk (E.L.W.); fbsajps@leeds.ac.uk (A.J.P.S.); \\ fbscpe@leeds.ac.uk (C.P.M.); r.m.chandler-bostock@leeds.ac.uk (R.C.-B.); A.M.Barker@leeds.ac.uk (A.M.B.) \\ 2 Departments of Biology and Mathematics \& York Cross-disciplinary Centre for Systems Analysis, \\ University of York, York YO10 5DD, UK; reidun.twarock@york.ac.uk (R.T.); sam.clark@york.ac.uk (S.C.); \\ r.j.bingham@york.ac.uk (R.J.B.) \\ * Correspondence: p.g.stockley@leeds.ac.uk; Tel.: +(44)-113-343-3092 \\ † Presented at Viruses 2020-Novel Concepts in Virology, Barcelona, Spain, 5-7 February 2020.
}

Published: 30 June 2020

\begin{abstract}
Single-stranded RNA viral genomes (gRNA) are dynamic molecules that permit packaging into virions and their subsequent extrusion during infection. For viruses with such genomes, we discovered a previously unsuspected mechanism that regulates their assembly. This regulation is the result of multiple cognate coat protein $(\mathrm{CP})$-gRNA contacts distributed across the RNA. Collectively, these interactions make the assembly highly efficient and specific. The regions of the gRNA packaging signals (PSs) driving this assembly are potential drug targets, whilst the manipulation of PS-CP contacts with nonviral RNA cargos is a route towards bespoke virus-like particles. Infectivity depends on the virions being able to transfer their gRNAs into host cells. The starting point for this transfer appears to be an encapsidated RNA with a defined three-dimensional structure, especially around the PSs. A combination of asymmetric cryo-electron microscopy structure determination and X-ray synchrotron footprinting were used to define these contacts and structures in a number of viral examples, including hepatitis B virus and enteroviruses. These tools allow us to look beyond the outer CP layer of the virion shell and to see the functional, asymmetric components that regulate viral infectivity. This revealed yet more unexpected aspects of critical infection mechanisms, such as the RNA conformational changes required for encapsidation, the details of PS-CP contacts regulating the assembly, and the conformational "memory" imposed by encapsidation.
\end{abstract}

Keywords: virus; ssRNA genome; packaging; assembly; packaging signals; mechanism

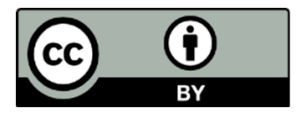

(c) 2020 by the authors. Licensee MDPI, Basel, Switzerland. This article is an open access article distributed under the terms and conditions of the Creative Commons Attribution (CC BY) license (http://creativecommons.org/licenses/by/4.0/). 